\title{
Beam Loss and Collimation at LHC
}

J.B. Jeanneret, O. Aberle, I.L. Ajguirei*,†, R. Assmann, I.S. Baichev*,†, J-P. Bojon, D. Kaltchev ${ }^{\ddagger}, \S$, L. Bruno, E. Carlier, E. Chapochnikova, E. Chiaveri, B. Dehning, S. Fartoukh, A. Ferrari, B. Goddard, J.M. Jimenez, V. Kain, I. Kourotchkine, $\dagger$, H. Preis, F. Ruggiero, R. Schmidt,P. Sievers, J. Uythoven, V. Vlachoudis, L. Vos, E. Vossenberg

\begin{abstract}
After a short review of past collimation work at LHC, the conception and baseline of the LHC collimation system are described. Abort-gap cleaning and beam loss monitoring are also discussed.
\end{abstract}

Paper presented at the HALO03 ICFA Advanced Beam Dynamic workshop, held at Montauk, USA, May 2003.

\footnotetext{
* Institute for High Energy Physics, Protvino, Russia.

${ }^{\dagger}$ Member of the Russian collaboration to the LHC Project.

¥TRIUMF, Vancouver, Canada

$\S$ Member of the Canadian collaboration to the LHC Project
} 


\title{
Beam Loss and Collimation at LHC
}

\author{
J.B. Jeanneret*, O. Aberle*, I.L. Ajguirei ${ }^{\dagger}$, R. Assmann*, I. Baishev ${ }^{\dagger}$, J-P. Bojon*, \\ L. Bruno*, E. Carlier*, E. Chapochnikova*, E. Chiaveri*, B. Dehning*, \\ S. Fartoukh*, A. Ferrari*, B. Goddard*, J.M. Jimenez*, D. Kaltchev**, V. Kain*, \\ I. Kourotchkine ${ }^{\dagger}$, H. Preis*, F. Ruggiero*, R. Schmidt*, P. Sievers*, J. Uythoven*, \\ V. Vlachoudis*, L. Vos* and E. Vossenberg* \\ ${ }^{*}$ CERN, Geneva, Switzerland \\ ${ }^{\dagger}$ IHEP, Protvino, Russia \\ ${ }^{* *}$ TRIUMF, Vancouver,Canada
}

\begin{abstract}
After a short review of past collimation work at LHC, the conception and baseline of the LHC collimation system are described. Abort-gap cleaning and beam loss monitoring are also discussed.
\end{abstract}

\section{INTRODUCTION}

A bit more than ten years ago, collimation in proton colliders was still in a kind of prehistory. Collimation was used mainly to limit the background to the experiments. This changed with the SSC and LHC projects, where stored beam energies opened a new scale for loss control and machine protection. Beam induced quenches became an issue which deserved a quantitative approach. The emphasis was then focused on the optics of a two-stage collimation system [9, 10]. For LHC, a long straight section was early on dedicated to the betatron collimation system $[1,2]$. Two codes were developed, STRUCT for SSC and K2 for LHC, in order to quantify precisely the residual losses associated to a collimation system [3, 4]. These codes combined scattering at the edge of a jaw and tracking around the ring. They allowed to compute beam loss densities along the ring in the presence of collimators. Using the cascade codes MARS and FLUKA $[5,6]$, peak energy and power densities were simulated and compared to quench limit calculations [7]. Low-Z materials were obvious good choices for collimator jaws. With their large radiation length they accept substantially higher rates than high- $Z$ ones. The best candidates were (and still are) graphite and beryllium. The first one was rejected for poor vacuum properties and a potential for dust release. Beryllium was discarded for toxicity reason, even if the use of massive blocks in vacuum is not really problematic. Eventhough as little as $5 \%$ of a proton bunch can already damage a piece of copper at 7 $\mathrm{TeV}$ in LHC in case of frontal impact, it was initially decided to use aluminum and copper, for primary and secondary collimators respectively. The dogma was to rely on safe and clever operation. It was considered that modern control systems would allow to detect early enough any drift away from safe predefined conditions. More

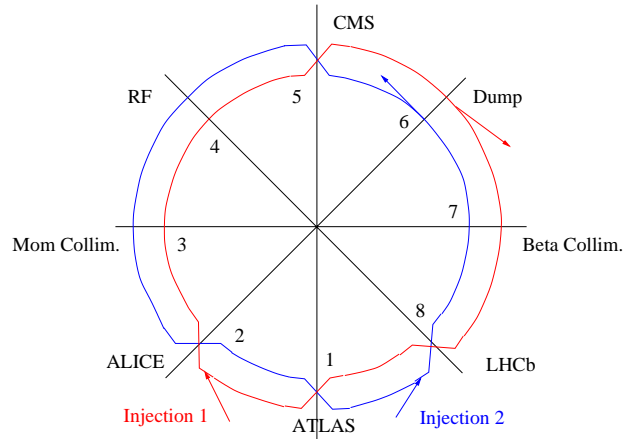

FIGURE 1. A schematic layout of the LHC ring

recently, after a review of the failure rate of the dump kicker error, which induce severe beam losses, the probabilities of such events was raised substantially. Then, operational and machine integrity issues were considered in a more balanced way. A substantial revision of the collimation system was therefore initiated recently $[11,12]$, together with the design of a structured machine protection scheme [13]. Finally, the use of additional single turn beam absorbers associated with the injection and dump systems is presently studied coherently with multiturn collimation [14].

\section{BASIC CONCEPTS}

In order to avoid the early dump of a beam during injection, ramping or steady collisions, it is mandatory to avoid a quench resulting from bad but not rare conditions. In Table 2, transient and steady losses are compared to quench limits [7]. The efficiency of collimation must be $\eta<10^{-4} \mathrm{~m}^{-1}$. The quantity $\eta$ is the rate of losses per meter of ring at an aperture limitation divided by the primary loss rate. This high efficiency requires the use of a two-stage collimation system [8]. The op- 
TABLE 1. Some LHC beam parameters.

\begin{tabular}{lrll}
\hline Luminosity & $10^{34}$ & $\mathrm{~cm}^{-2} \mathrm{~s}^{-1}$ & \\
$\sigma^{*}$ at crossing & 16 & $\mu \mathrm{m}$ & $\beta^{*}=0.5 \mathrm{~m}$ \\
Nominal bunch & $1.0510^{11}$ & protons & \\
Stored beam & $310^{14}$ & protons & 2800 bunches \\
Beam energy & 450 & $\mathrm{Gev}$ & Injection \\
Beam energy & 7000 & $\mathrm{Gev}$ & Collision \\
Injected energy & $210^{6}$ & $\mathrm{~J}$ & $24 \times 4 \mathrm{~kg} \mathrm{Cu}$ \\
Stored energy & $34010^{6}$ & $\mathrm{~J}$ & $2 \times 800 \mathrm{~kg} \mathrm{Cu}$ \\
Loss rate & $310^{9} \mathrm{p} / \mathrm{s}$ & $3 \mathrm{~kW}$ & $\tau_{\text {beam }}=30 \mathrm{hr}$ \\
Peak loss rate & $10^{11} \mathrm{p} / \mathrm{s}$ & $100 \mathrm{~kW}$ & $\tau_{\text {beam }} \sim 1 \mathrm{hr}$ \\
\hline
\end{tabular}

* Melted copper

TABLE 2. Expected regular transient (top part of the table) and steady (bottom part) losses compared to quench limits. At injection we consider the loss of 5\% of an injected batch, associated with an error of the damping of the injection oscillation. At ramping, $10 \%$ of the beam may be off-bucket because of RF phase errors. At collision, degraded conditions, which require some time to be corrected, may lower the beam-lifetime down to $\tau=1$ hour. All these values are indicative.

\begin{tabular}{lrrr}
\hline Case & $\Delta N[\mathrm{p}]$ & $\Delta N_{q}[\mathrm{p} / \mathrm{m}]$ & $\eta=\Delta N_{q} / \Delta N[1 / \mathrm{m}]$ \\
\hline Injection & $1.2510^{12}$ & $10^{9}$ & $810^{-4}$ \\
Ramping & $310^{13}$ & $2.510^{10}$ & $810^{-4}$ \\
\hline & $\dot{N}[\mathrm{p} / \mathrm{s}]$ & $\dot{N}[\mathrm{p} / \mathrm{m} / \mathrm{s}]$ & $\eta=\dot{N}_{q} / \dot{N}$ \\
\hline $7 \mathrm{TeV}$ & $810^{10}$ & $810^{6}$ & $10^{-4}$ \\
\hline
\end{tabular}

tics of a two-stage two-dimensional collimation system is designed by considering that protons scattered out of a collimator occupy the whole ( $\left.\mathrm{x}^{\prime}, \mathrm{y}^{\prime}\right)$ space, even if large values are unlikely. This 'stochastic coupling' imposes the use of several secondary jaws per primary collimator (in LHC four of them), in order minimize the size of the secondary halo. In a true 2D-2-stage collimation system, an optimum is obtained with well defined correlated transverse betatron phase advances between primary and secondary collimators [10]. If the primary aperture defined by the primary jaws is $n_{1} \sigma_{\beta}$ and the secondary one defined by the secondary jaws is $n_{2} \sigma_{\beta}$, all the phases and the normalized skew angles of the jaws are expressed with either the angle $\mu_{o}=\cos ^{-1}\left(n_{1} / n_{2}\right)$ or a rational fraction of $\pi$, see Table 3 . At ramping, momentum collimation must be used $[9,10]$. Conflicting optics requirements imply to use separate insertions for betatron and momentum collimation even if their optics are similar $[10,17]$. Figure 1 shows the location of the cleaning insertions in the LHC ring. In an insertion of finite length, the best correlation of the phase advances can never be reached. It can at best be optimized. The location and the transverse tilt of the jaws are calculated numerically $[15,16]$. The optics of the two insertions of LHC are discussed in [17]. They are presently revised, in order to satisfy new requirements related to impedance considerations, see Section 6.
TABLE 3. Correlated phase advances between primary and secondary jaws $\mu_{x}$ and $\mu_{y}$ and $X-Y$ jaw orientations $\alpha_{\text {Jaw }}$ for three primary jaw orientations $\alpha$ and four scattering angles $\phi$ with $\mu_{o}=$ $\cos ^{-1}\left(n_{1} / n_{2}\right)$.

\begin{tabular}{ccccc}
$\alpha$ & $\phi$ & $\mu_{x}$ & $\mu_{y}$ & $\alpha_{\text {Jaw }}$ \\
\hline 0 & 0 & $\mu_{o}$ & - & $0^{*}$ \\
0 & $\pi$ & $\pi-\mu_{o}$ & - & $0^{*}$ \\
0 & $\pi / 2$ & $\pi$ & $3 \pi / 2$ & $\mu_{o}{ }^{*}$ \\
0 & $-\pi / 2$ & $\pi$ & $3 \pi / 2$ & $-\mu_{o}{ }^{*}$ \\
$\pi / 4$ & $\pi / 4$ & $\mu_{o}$ & $\mu_{o}$ & $\pi / 4$ \\
$\pi / 4$ & $5 \pi / 4$ & $\pi-\mu_{o}$ & $\pi-\mu_{o}$ & $\pi / 4$ \\
$\pi / 4$ & $3 \pi / 4$ & $\pi-\mu_{o}$ & $\pi+\mu_{o}$ & $\pi / 4$ \\
$\pi / 4$ & $-\pi / 4$ & $\pi+\mu_{o}$ & $\pi-\mu_{o}$ & $\pi / 4$ \\
$\pi / 2$ & $\pi / 2$ & - & $\mu_{o}$ & $\pi / 2$ \\
$\pi / 2$ & $-\pi / 2$ & - & $\pi-\mu_{o}$ & $\pi / 2$ \\
$\pi / 2$ & $\pi$ & $\pi / 2$ & $\pi$ & $\pi / 2-\mu_{o}$ \\
$\pi / 2$ & 0 & $\pi / 2$ & $\pi$ & $\pi / 2+\mu_{o}$ \\
\hline
\end{tabular}

* Also used for momentum collimation

\section{JAW MATERIALS}

A dump kicker error at top energy is the worst case for damage of a jaw. For quite a time, it was believed that the probability of such an event was larger than 20 years ${ }^{-1}$. It was therefore considered that the small risk of destruction of a collimator was acceptable. This probability was revised two years ago to $\sim 1$ year $^{-1}$ and the collimator jaws had to be redesigned to survive to this kind of events. The choice of materials discussed in Section 1 was therefore revised. Two kinds of dump kicker errors may occur. An external spurious trigger may fire the whole set of 15 kicker modules, or one module may auto-trigger [18]. In both cases the beam is spread quite uniformly between the ring beam axis and the dump channel axis. In the latter case, the other modules must be re-triggered rapidly, in order to avoid that most of the circulating beam is kicked inside the aperture of the ring. The former re-triggering time was $\tau_{\text {re-trig }}=1.3 \mu \mathrm{s}$, with 20 bunches impacting a jaw. Even the best species of graphite would suffer some damage. The internal dump timing system was revisited and now offers $\tau_{\text {re-trig }}=0.7 \mu \mathrm{s}$, with the impact of 8 bunches only, see table 4. FLUKA [6] and ANSYS simulations indicate that good species of graphite can stand this impact. The second best material is beryllium. Because of its large Young modulus and in spite of its smaller atomic number, it is already eight times worse than graphite, in terms of ultimate tensile strength, see table 4 . Beryllium can be envisaged only for those jaws which cannot be touched by dump kicked beam (approximately a half of them) while some injection failure modes remain to be fully explored. All other materials must be discarded, see Table 5. An absorber (TCDQ) must be installed behind 
TABLE 4. Number of bunches impacting the collimator jaws in case of dump errors. The quantity $\tau_{\text {re-trig }}$ is discussed in the text. SDF stands for 'survival deficit factor', a value which is related to the ultimate tensile strength. A good case is $\mathrm{SDF}<1$.

\begin{tabular}{lrcr}
\hline Case & Nb. bunches & \multicolumn{2}{c}{ SDF } \\
& on jaw & Graphite & Beryllium \\
\hline Auto-trigger & & & \\
$\tau_{\text {re-trig }}=1.3 \mu \mathrm{s}$ & 20 & 1.6 & 12 \\
$\tau_{\text {re-trig }}=0.7 \mu \mathrm{s}$ & 8 & 0.7 & 5 \\
\hline External trigger & 5 & 0.4 & 3 \\
\hline
\end{tabular}

TABLE 5. Radiation length and temperature increase after impact of 8 nominal bunches spread between 5 and $10 \sigma_{\beta}$ on a jaw for some materials.

\begin{tabular}{lrrr}
\hline Material & $Z$ & $X_{0}[\mathrm{~cm}]$ & $\Delta T_{\max }\left[{ }^{\circ} \mathrm{C}\right]$ \\
\hline Beryllium & 4 & 35.3 & 310 \\
Graphite & 6 & 18.8 & 800 \\
Aluminum & 13 & 8.9 & 2700 \\
Titanium & 22 & 3.6 & $\geq 5000$ \\
Copper & 29 & 1.4 & $\geq 5000$ \\
\hline
\end{tabular}

the dump kickers at a phase advance of $\mu=\pi / 2$ and at a depth of $\sim 10 \sigma_{\beta}$, in order to protect the low- $\beta$ insertions which are located in between the dump area and the collimation insertion [19], see Fig. 1. The TCDQ will be thick enough to avoid damages of the nearby machine elements. In case of kicker error, similar beam densities will impact the TCDQ and the collimators. The former one too will thus be made of graphite. The vacuum problems expected with graphite have been studied recently. A dump device made of graphite was installed in the SPS ring, and revealed no dust release. New treatments of graphite were worked-out [20, 21]. Initial outgassing is dominated by long hydrocarbon chains, which are residues of materials used during the compaction process of the graphite block. In order to improve its vacuum properties, a heat treatment at $1000{ }^{\circ} \mathrm{C}$ burns and expels most of these chains. Later regular in-situ bakeout at $300^{\circ} \mathrm{C}$ exhibits the usual low-atomic mass spectra. Out-gassing rates are quite similar to usual metallic vacuum components.

\section{ABORT GAP CLEANING}

Apart from the case of ramping, where uncaptured protons induce a flash of losses soon after the beginning of the ramp $[22,23]$, momentum collimation is needed to capture off-bucket protons which loose momentum by synchrotron radiation at top energy [24]. Long storage time of particles with large momentum offset must be avoided. Their detuning with momentum can be quite large (the momentum aperture of the ring is $\approx 6 \times 10^{-4}$ )

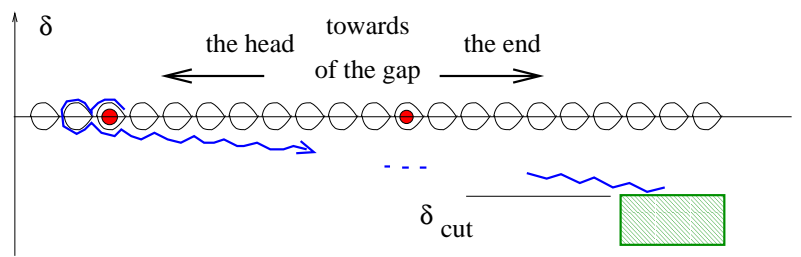

FIGURE 2. The longitudinal motion of a proton which left the bucket. It looses momentum by synchrotron radiation and is finally captured by the primary momentum collimator.

and thus the effective aperture may differ from the nominal one for these. In addition, these particles creep along the bunch structure and invade the abort gap. If their density is too large there, a quench will occur in the magnets downstream the dump system even during regular dump actions. The phenomenon is similar to the dump error dicussed in Section 3. A detailed description of this effect is in preparation [25] and is illustrated in Figure 2. The peak density in the abort gap is given here by the very simplified expression

$$
\hat{\rho}_{0} \simeq 0.7 \frac{N_{0}}{\tau_{\text {long }} L_{\text {ring }}} \frac{\delta_{\text {cut }}}{\dot{\delta}}=2.210^{7} \mathrm{p} / \mathrm{m},
$$

with $N_{0}$ the number of stored protons (see Table 1), $\tau_{\text {long }}=10 \mathrm{~h}$ a somewhat low longitudinal beam lifetime, $L_{\text {ring }}=26660 \mathrm{~m}, \dot{\delta}_{\text {cut }} \simeq 10^{-3}$ the momentum cut made by the momentum collimation system at top energy and $\dot{\delta}=U_{0} f_{r} / E_{\text {beam }}=10^{-5}$ the momentum loss per second by synchrotron radiation with $U_{0}=7 \mathrm{keV} / \mathrm{turn}$, $E_{\text {beam }}=7 \mathrm{TeV}$ and $f_{r}=1.110^{4} \mathrm{~Hz}$ the rotation frequency. The coefficient 0.7 is obtained by the integration of the synchrotron motion between $\delta_{\text {bucket }}$ and $\delta_{\text {cut }}$ and by summing over all occupied buckets. This value is case specific and shall be used only indicatively, see [25] for a complete formalism. The peak density is reached at the rear side of the abort gap, because particles with negative $\delta_{p}$ creep forward. In our case, the density at the head is $\rho_{\text {head }} \approx \hat{\rho}_{0} / 2=1.2 \times 10^{7} \mathrm{p} / \mathrm{m}$. This value is larger than the critical $\rho_{\text {tol }} \approx 0.4 \times 10^{7} \mathrm{p} / \mathrm{m}$, above which a quench is induced behind the dump system [19]. It is intended to make use of the transverse damper, used in an excitation mode, in order to grow the betatronic amplitude of the particles which are present in the abort gap, and thus accelerate their capture. It is very fortunate that the dangerous part of the abort gap is located at its head (this is where the dump kicker starts to rise and sprays the beam at low amplitude). The creeping protons must traverse the entire gap before reaching the head. This allows to let the damper work mostly in the central part of the gap, leaving enough time for turning on and off the excitation mode. 


\section{BEAM LOSS MONITORING}

In addition to detectors which will be installed all along the ring and in every critical location, in particular near experiments, beam loss monitors will be installed near every collimator [26]. The monitors will be connected to the dump trigger system and used to check on-line if the collimation setting is correct. They may also be used, to some extent, to diagnose damage of the collimator jaws. The data recorded near collimators will not be easy to use and interpret. At high energy, the cascade developed in a jaw and in the surrounding material will induce a signal in all the monitors which are installed nearby and downstream of it. In order to understand how to use the signals, we made a preparatory simulation with MARS, which develops cascades into the entire momentum cleaning section ( 7 collimators and monitors), including vacuum chambers, magnets with their field, tunnel, ground, etc [27]. A primary impact map was generated with K2. The partial fluences as issued from every collimator were recorded at each monitor, allowing to build a matrix which allows to compute the normalized rate $s_{i}$ at every monitor as a function of the primary rate $r_{i}$ at each collimator. For the nominal working condition at injection energy, for $\vec{s}=\mathbf{M} \vec{r}, \mathbf{M}$ is equal to

$\begin{array}{|lllllll|}.0178 & .0 & .0 & .0 & .0 & .0 & .0 \\ .4662 & 1.19 & .0 & .0 & .0 & .0 & .0 \\ .0268 & .0291 & 1.081 & .0004 & .0 & .0 & .0 \\ .0432 & .0389 & 1.085 & 1.044 & .0 & .0 & .0 \\ .0079 & .0036 & .138 & .3245 & .9891 & .0 & .0 \\ .0036 & .0017 & .03858 & .1187 & .513 & .9848 & .0 \\ .0012 & .0007 & .0099 & .0349 & .1642 & .5093 & .9445\end{array} \mid$

Further work will include a variation of the jaw depth $n_{i}$ one by one, in order to map $\mathbf{M}$ as a function of $\vec{n}$. With beam, $\mathbf{M}$ may be constructed by sending a pilot bunch on every jaw one after one. With the high value of many non-diagonal terms in $\mathbf{M}$, we are not yet sure that unambiguous calculations of the loss rate on every collimator can be deduced with this approach. It may be necessary to add more counters, in order to be overdeterministic and to remove ambiguities.

\section{BASELINE}

A new baseline design was defined recently. It deserves further detailed studies and discussion. It is just outlined here. In phase 1, during the early period of operation with relaxed beam parameters (half stored current, in particular), the jaws will be made of graphite. They will not only survive dump errors, but allow for quite degraded lifetime conditions. At $7 \mathrm{TeV}$, the resistive $Z_{\perp}$ will be too high with all the secondary jaws at $n_{2}=7$. With $n_{2}=10$, and $n_{1}=[6-8]$, yielding a secondary halo size of $A_{\mathrm{sec}} \approx 13 \sigma_{\beta}, Z_{\perp} \sim 330 \mathrm{M} \Omega / \mathrm{m}$. This value is compatible with the damping strength of the Landau octupoles. The aperture of the experimental triplet must be $A_{\text {triplet }} \approx A_{\text {sec }}$, and therefore $\beta^{*} \approx 0.85 \mathrm{~m}$. The luminosity will thus be slightly reduced during this phase. The penetration of the EM fields in the graphite will dissipate $P \sim 400 \mathrm{~W}$ in the $1 \mathrm{~m}$-long secondary jaws. A water cooling of the jaw is therefore mandatory, even in the abscence of direct beam power deposition. In phase 2, in order to to reach the nominal performance, $n_{2}$ will be reduced by replacing some graphite jaws (graphite with thin film coating, graphite with higher conductivity, beryllium or other good conductors). Operational experience will tell us which jaws are not subject to impact following kicker errors and in the meantime, these alternative will be explored and tested.

\section{REFERENCES}

1. Design study of the Large Hadron Collider, Chapter 9, CERN/91 -03, 1991.

2. J.B. Jeanneret, CERN SL/EA/Note 90-01, 1990.

3. I. Baichev, et al., SSCL-MAN-0034, Dallas, 1994.

4. T. Trenkler and J.B. Jeanneret, CERN SL/Note 94-105 (AP), 1994.

5. I.L.Azhgirey, I.A.Kurochkin and V.V.Talanov, in: Ann. of XV Conf. on Charg. Part. Accel., p.74, Protvino, 22-24.10.96 (in russian).

6. A. Fasso et al., Proc. Monte-Carlo 2000 Conf., Lisbon, Springer-Verlag Berlin, p. 955, 2000.

7. J.B. Jeanneret et al., CERN LPR 44, 1996.

8. The Large Hadron Collider, CERN/AC/95-05(LHC), Chap.4,1995.

9. T. Trenkler and J.B. Jeanneret, Part.Acc., 50 , (1995) 287, January 1995.

10. J.B. Jeanneret, Phys.Rev. ST-AB, 1, 081001, Dec. 1998.

11. R. Assmann et al., CERN LPR 599 and EPAC02, 2002.

12. R. Assmann et al., CERN LPR 640 and PAC03,2003.

13. R. Schmidt, these proceedings.

14. H. Burkhardt, B. Goddard and V. Mertens, CERN LPR 641 and PAC03,2003.

15. D.I. Kaltchev et al., CERN LPR 134 and PAC1997,1997.

16. D.I. Kaltchev et al., CERN LPR 194 and EPAC98,1998.

17. D.I. Kaltchev et al., CERN LPR 305 and PAC1999,1999.

18. R. Assmann, B. Goddard, E. Vossenberg and E. Weisse, CERN LPN 293,2002.

19. A.I. Drozhdin et al., Fermilab Project Note FN-0724,2002.

20. J-P. Bojon et al., CERN AT Note, to be issued,2003.

21. P. Chiggiato, Private communication, 2002.

22. J.B. Jeanneret, SL/Note 92-56 (EA), 1992.

23. I. Baishev et al., CERN LPR 309,1999.

24. J.B.Jeanneret, CERN/SL/92-44(EA).

25. E. Chapochnikova,S. Fartoukh and J.B. Jeanneret, CERN LPN, to be issued 2003.

26. J.B.Jeanneret et al., CERN LHC-BLM-ES-0001.00 rev 1.1, EDMS doc 328146, 2003.

27. I. Ajguirei, I.S. Baichev, J.B. Jeanneret and I.A. Kourotchkine, CERN LPN, to be issued, 2003. 